\title{
Combining Transcranial Direct Current Stimulation and Transcutaneous Electrical Nerve Stimulation to Relieve Persistent Pain in a Patient Suffering from Complex Regional Pain Syndrome: A Case Report
}

This article was published in the following Dove Press journal:

Journal of Pain Research

\author{
Francis Houde ${ }^{1-3}$ \\ Marie-Philippe Harvey ${ }^{1,3}$ \\ Pierre-François Tremblay \\ Labrecque (D) 1,3 \\ Francis Lamarche ${ }^{1,3}$ \\ Alexandra Lefebvre ${ }^{1,3}$ \\ Guillaume Leonard (1D) \\ 'Research Center on Aging, CIUSSS de \\ l'Estrie - CHUS, Université de \\ Sherbrooke, Sherbrooke, Québec, \\ Canada; ${ }^{2}$ Research Center of the Centre \\ hospitalier universitaire de Sherbrooke \\ (CHUS), CIUSSS de l'Estrie - CHUS, \\ Université de Sherbrooke, Sherbrooke, \\ Québec, Canada; ${ }^{3}$ Faculté de Médecine \\ et des Sciences de la Santé, Université de \\ Sherbrooke, Québec, Canada
}

Correspondence: Guillaume Leonard Centre de recherche sur le vieillissement, CIUSSS de l'Estrie - CHUS, 1036, rue Belvédère Sud, Sherbrooke, Québec JIH 4C4, Canada

Tel +I (8I9) 829-7I3I ext. 45246

$\mathrm{Fax}+\mathrm{I}$ (8I9) 820-6864

Email guillaume.leonard2@usherbrooke.ca
Purpose: Complex regional pain syndrome (CRPS) is a rare neuropathic pain condition characterized by sensory, motor and autonomic alterations. Previous investigations have shown that transcranial direct current stimulation (tDCS) and transcutaneous electrical nerve stimulation (TENS) can alleviate pain in various populations, and that a combination of these treatments could provide greater hypoalgesic effects. In the present case report, we describe the effect of tDCS and TENS treatment on pain intensity and unpleasantness in a patient suffering from chronic CRPS.

Results: The patient was a 37-year-old woman, suffering from left lower limb CRPS (type I) for more than 5 years. Despite medication (pregabalin, tapentadol, duloxetine), rehabilitation treatments (sensorimotor retraining, graded motor imagery) and spinal cord stimulation (SCS), the participant reported moderate to severe pain. Treatments of tDCS alone (performed with SCS turned off during tDCS application, 1 session/day, for 5 consecutive days) did not significantly decrease pain. Combining tDCS with TENS (SCS temporarily turned off during tDCS, 1 session/day, for 5 consecutive days) slightly reduced pain intensity and unpleasantness.

Discussion: Our results suggest that combining tDCS and TENS could be a therapeutic strategy worth investigating further to relieve pain in chronic CRPS patients. Future studies should examine the efficacy of combined tDCS and TENS treatments in CRPS patients, and other chronic pain conditions, with special attention to the cumulative and long-term effects and its effect on function and quality of life.

Keywords: chronic pain, neuropathic pain, electrotherapy, peripheral electrical stimulation, peripheral nerve stimulation, non-invasive brain stimulation

\section{Introduction}

Complex regional pain syndrome (CRPS) is a rare neuropathic pain condition, characterized by sensory, motor and autonomic alterations, which typically occur following an injury. ${ }^{1-5}$ CRPS is characterized by continuous and disproportionate pain relative to the initial event and can be subdivided into two categories, based on the absence (type I) or presence (type II) of a peripheral nerve lesion. ${ }^{3,6}$ The exact pathogenesis of CRPS remains elusive, even though growing evidences suggest that many factors (including neurogenic inflammation, autonomic dysregulation and maladaptive neuroplasticity) are implicated in this painful disorder. ${ }^{4,7-9}$ Unfortunately, at this point, no 
clear evidence-based approaches are currently accepted in the treatment of CRPS. ${ }^{10,11}$ However, practical guidelines suggest that pain management and physical rehabilitation should begin as soon as possible after a surgery. Avoiding immobilization and fostering a rapid return to normal function of the limb could help to prevent and manage early CRPS. $^{4,12-15}$

Transcranial direct current stimulation (tDCS) is a promising non-invasive brain stimulation technique that has been proven useful in various chronic pain syndromes that are refractory to conventional treatments. ${ }^{5,16-19}$ Although not fully understood, analgesic effects of tDCS are thought to be driven by cortical excitability modulation, and possibly by endogenous $\mu$-opioids release when applied over the motor cortex. ${ }^{20-24}$ Another interesting non-pharmacological approach used in pain rehabilitation is transcutaneous electrical nerve stimulation (TENS). ${ }^{25-27}$ Low-frequency TENS $(<10 \mathrm{~Hz})$ activates descending pain inhibition systems (conditioned pain modulation [CPM]) and promotes the release of endogenous opioid mechanisms that can markedly reduce pain symptoms. ${ }^{27-32}$ The combination of tDCS and TENS have been proposed by previous authors, due to their potential synergetic effect on pain. ${ }^{33-35}$ Boggio and colleagues have shown that combining tDCS with TENS is more effective than tDCS alone in individuals suffering from neurogenic pain affecting the upper extremities. ${ }^{33}$ More recently, Schabrun and colleagues observed that a combined tDCS/TENS intervention was superior to either technique used alone for patients suffering from chronic low back pain. ${ }^{34}$ These results led us to believe that tDCS (alone and in combination with TENS) could be an effective treatment for a CRPS patient with persistent symptoms who experienced an important and unexpected exacerbation of her pain, which could not be relieved using usual care.

\section{Case Description}

A 32-year-old woman was diagnosed with CRPS Type I (Budapest criteria) ${ }^{3}$ by an anesthesiologist, three weeks after hitting here left lower limb on a piece of furniture. She had disproportionate pain compared to the inciting event (continuous moderate to severe pain on a daily basis after the event), and exhibited typical CRPS symptoms and clinical signs at the moment of the evaluation, including 1) somatosensory (allodynia, hyperalgesia and agraphesthesia from toes to mid-thigh), 2) vasomotor (lower skin temperature and change in skin color of the affected foot and leg), 3) sudomotor (edema) and 4) motor/trophic (dystonia in ankle plantar flexion and increased nail growth) manifestations. Movements from her left foot and ankle were completely absent, constraining her to use Canadian crutches to move since the accident. She was prescribed pregabalin $150 \mathrm{mg} /$ day (once a day [DIE]), tapentadol $150 \mathrm{mg}$ (twice a day [BID]) and duloxetine $60 \mathrm{mg}$ DIE for pain relief.

Approximately one year after the initial incident, the patient began rehabilitation treatments including sensorimotor retraining and graded motor imagery 2-3 times a week for 16 weeks. ${ }^{36,37}$ Rehabilitation slightly decreased pain and reduced the area of allodynia and hyperalgesia as far as mid-tibia but had no effect on motor symptoms. Two years after the trauma, the patient was surgically implanted with a spinal cord stimulator (SCS), which significantly reduced her pain, to the point that she was now able to touch (mechanical stimuli) her affected limb and put on socks and shoes over her left foot. Subsequently, non-painful stimulations with low-frequency TENS applied directly on the lower leg of the affected limb were added as daily home treatments to stimulate afferent fibers in order to promote cerebral plasticity and motor recovery. ${ }^{38-40}$ After 8 weeks of TENS, the patient was able to slightly move her affected ankle for the first time in 2 years. Even though pain never completely disappeared, the condition of the patient remained stable (mean pain intensity of approximately 3 / 10 on a visual analog scale [VAS]) for another two and a half years until the patient (now aged 37 years) reported a significant worsening of her symptoms (increased pain and reduced voluntary movement of the foot and ankle). No events occurred before the worsening of symptoms, and pharmacological analgesics and SCS were pursued as usual, although they suddenly appeared to be ineffective. Previous rehabilitation treatments (graded motor imagery and sensorimotor training) were tried once again, without success.

\section{Intervention}

The ethics committee of the Research Center on Aging approved the intervention protocol. The patient first received 5 sessions of tDCS alone (1 session/day for 5 consecutive days; Treatment A) without much results on her pain. Based on the promising results of Boggio et al and Schabrun et al, tDCS and TENS were concomitantly applied for 5 sessions (1 session/day, for 5 consecutive days, Treatment B) the next week. Six months later, combined tDCS and TENS treatments ( 1 session/day, for 5 consecutive days, Treatment C) were performed for a second time. tDCS was given with a constant current stimulator (NeuroConn Medical 
Technology, Ilmenau, Germany) used to transfer direct current to a pair of saline-soaked sponge electrodes $(5 \times 7 \mathrm{~cm})$. The center of the active electrode (anode) was placed over the right primary motor cortex ( $\mathrm{C} 4$ according to the electroencephalogram 10/20 system) and the reference electrode (cathode) was placed horizontally over the contralateral supraorbital region (over the left eyebrow). ${ }^{33,34,41,42}$ A constant current of $2 \mathrm{~mA}$ was applied for $25 \mathrm{~min}$. The current was ramped-up (from $0 \mathrm{~mA}$ to $2 \mathrm{~mA}$ ) and rampeddown (from $2 \mathrm{~mA}$ to $0 \mathrm{~mA}$ ) over $30 \mathrm{sec}$ at the beginning and at the end of the stimulation session, to avoid discomfort. For safety reasons, SCS was turned off prior to each tDCS and tDCS/TENS session and turned on again, after the end of each session.

Low-frequency TENS ( $3 \mathrm{~Hz}, 400 \mu \mathrm{s})$ was given using an Empi, Eclipse+ Digital device (Minnesota, USA). TENS stimulations were applied for $25 \mathrm{~min}$ with two pairs of electrodes, disposed over the mid-thigh of the affected limb just above the painful region (region exempted of allodynia/hyperalgesia) and the anterior leg of the unaffected limb. ${ }^{43}$ Application of electrodes directly on the affected lower limb was avoided given the allodynic manifestations in this area. The intensity of TENS on each pair of electrodes was adjusted independently every $5 \mathrm{~min}$ to obtain strong/noxious sensations to trigger counterirritation (diffuse analgesic effect) via descending pain inhibitory controls. It is important to note that previous TENS intervention used at home by the patient (weakmoderate intensity low-frequency TENS applied close to the allodynic region) aimed to foster cerebral plasticity and motor recovery, as opposed to this TENS intervention (high-intensity low-frequency TENS applied over remote body regions) which aimed to reduce pain. ${ }^{32,38,39,44}$

\section{Outcomes}

Pain intensity and pain unpleasantness measures were obtained using a VAS $(0-10 \mathrm{~cm} ; 0=$ no pain/not unpleasant, $10=$ worst pain imaginable/extremely unpleasant, respectively) to evaluate the short-term effect of the interventions. The patient was asked to evaluate the intensity and unpleasantness of her clinical pain: 1) before the intervention, 2) immediately after the intervention and 3) 15 min after the intervention (Figure $1 \mathrm{~A}$ and B). As can be seen from these figures, short-term effects on pain were absent or modest, at the most, although the combination of tDCS with TENS appeared to be slightly more effective than tDCS alone.

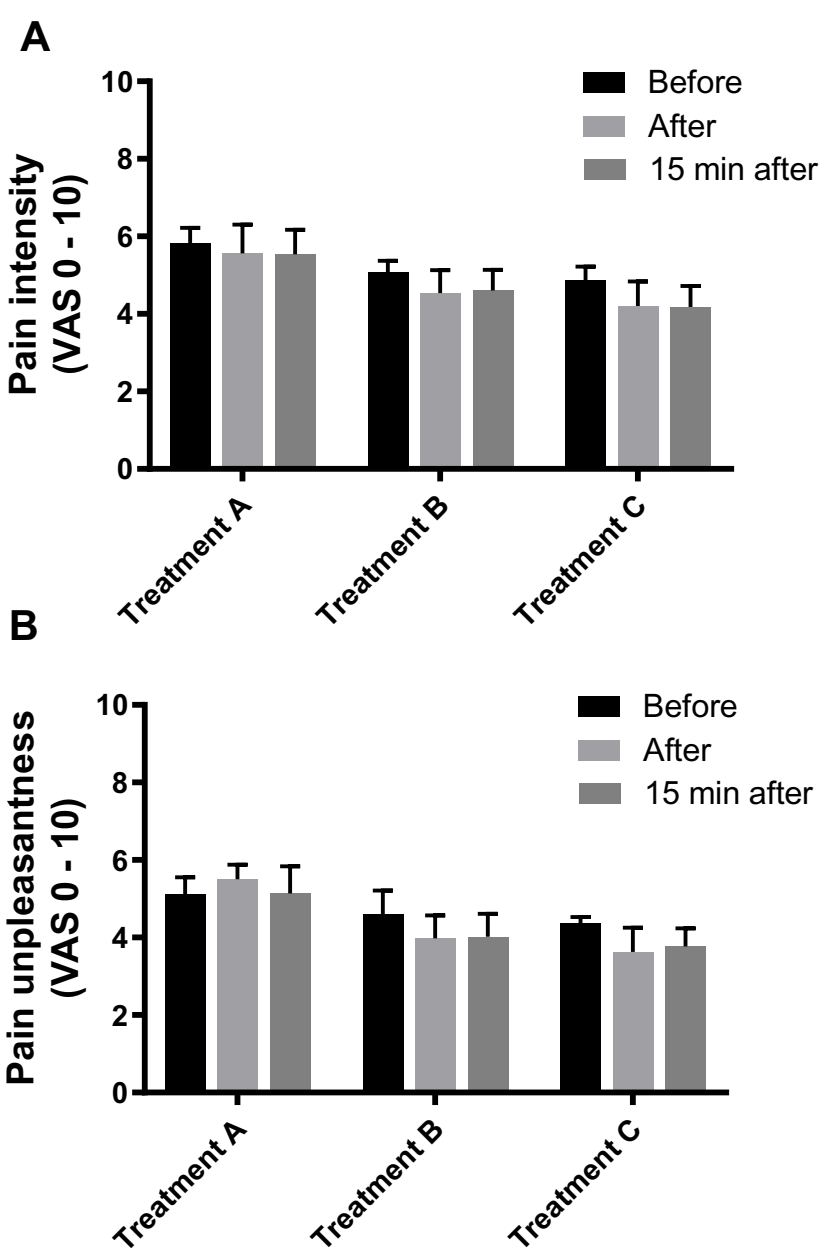

Figure I Pain intensity (A) and pain unpleasantness (B) scores obtained before, immediately after and 15 mins after tDCS alone (Treatment A); combined tDCS and TENS (Treatment B); and combined tDCS and TENS (Treatment $\mathrm{C}-6$ months later). Abbreviations: VAS, visual analog scale; tDCS, transcranial direct current stimulation; TENS, transcutaneous electrical nerve stimulation.

Long-term effects on pain were evaluated using 2 pain logbooks of 21 evaluation days each. Mean pain intensity (reflecting the average pain intensity felt during the day) was assessed in each logbook with a numerical rating scale (NRS; $0=$ no pain, $10=$ worst imaginable pain) at home, at the end of each day. The first logbook gathered information on the two first treatments ( 5 days of tDCS alone [Treatment A] and 5 days of tDCS combined with TENS [Treatment B, given the next week]) and a second logbook gathered data on the last intervention ( 5 days of tDCS combined with TENS [Treatment C]), given 6 months later (see Figure 2). Since the number of treatments evaluated differs between the two logbooks, the repartition of the 21 evaluation days also differs in each logbook (see horizontal axis of Figure 2). The first logbook evaluations allowed us to collect daily pain scores 1) seven days before (Pre-Tx), 2) five days during 


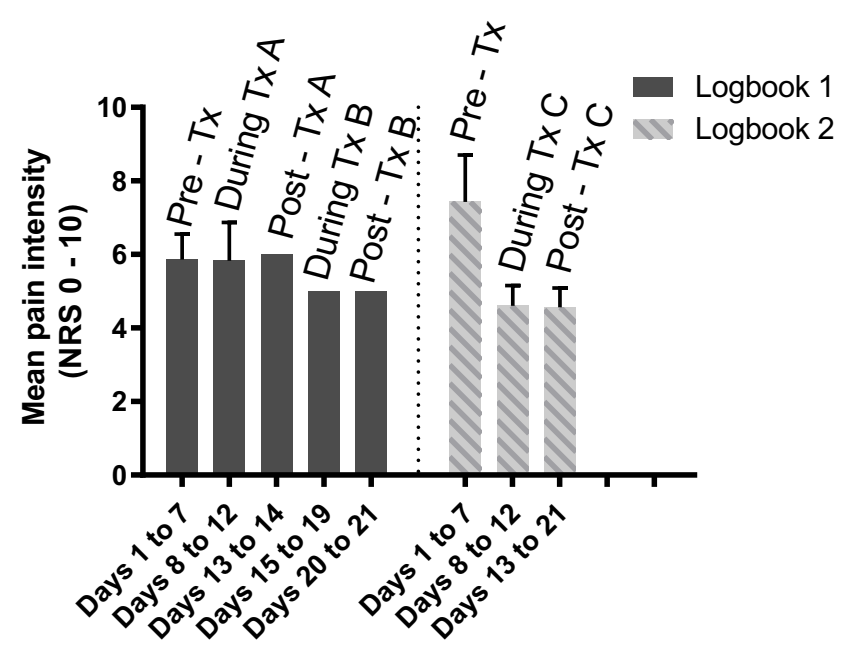

Figure 2 Mean pain intensity scores obtained with pain logbooks before, during and after interventions. Logbook I includes pain score values obtained before Treatment A (Pre-Tx A; days I-7), during Treatment A (During Tx A, tDCS alone; days 8-12), after Treatment A (Post-Tx A; days 13-14), during Treatment B (During Tx B, combined tDCS and TENS; days 15-19) and after Treatment B (Post-Tx B; days 20-2I). Logbook 2 includes pain score values obtained 6 months later, before Treatment C (Pre-Tx C; days I-7), during Treatment C (During Tx C, combined tDCS and TENS; days 8-12) and after Treatment C (Post-Tx C; days 13-21).

Abbreviations: NRS, numerical rating scale; TENS, transcutaneous electrical nerve stimulation; $\mathrm{tDCS}$, transcranial direct current stimulation.

tDCS alone (During Tx A), 3) two days between Treatment A and Treatment B (Post - Tx A), 4) five days during combined tDCS and TENS (During Tx B) and 5) two days after Treatment B (Post-Tx B), for a total of 21 days. Six months later, the second logbook assessments allowed us to collect daily pain scores 6) seven days before (Pre-Tx), 7) five days during combined tDCS and TENS (During Tx C) and 8) nine days after Treatment $\mathrm{C}$ (Post-Tx C), also for a total of 21 days.

As depicted in Figure 2, the application of tDCS alone did not affect daily pain ratings. The combination of tDCS and TENS appeared to be more effective than tDCS alone, even though only Treatment $\mathrm{C}$ reached clinical significance (reduction of $\geq 2$ points on the NRS). ${ }^{45,46}$ Pain reduction observed after Treatment $\mathrm{C}$ was greater than for Treatment B, suggesting a potential synergistic and/or cumulative effect of the interventions. Importantly, the pain reduction lasted at least 9 days after the last intervention (Treatment $\mathrm{C}$ ) as reported in the second pain logbook, even though the patient's pain never reached the pain levels reported before the exacerbation of her pain prior to our interventions (approximately $3 / 10$ on the VAS).

\section{Discussion}

In this case report, we tested if tDCS alone or in combination with TENS could be an effective strategy to relieve pain of our patient suffering from CRPS. For this patient, the combination of tDCS and TENS appears to be slightly more effective when compared to tDCS alone. These results are in line with previous investigations that observed greater effectiveness of the combination of tDCS and TENS modalities compared to both modality used alone in patients suffering from chronic neuropathic pain and chronic low back pain. ${ }^{33,34}$ More studies are required before any conclusions can be made regarding the effectiveness of this approach for CRPS patients, as our results describe the case of one patient only.

Our results pertaining to the short-term effects of our treatments indicate that tDCS (alone or in combination with TENS) did not have immediate effects on pain intensity and unpleasantness (see Figure 1A and B). One possible explanation for the absence of short-term effects for the tDCSTENS combination is the use of tapentadol (a $\mu$-opioid agonist) by the patient. Past studies have shown that the hypoalgesic effect of low-frequency TENS is substantially reduced in individuals who take $\mu$-opioid agonists on a regular basis; a phenomenon probably due to a crosstolerance effect between these two interventions, which both depend on the activation of $\mu$-opioid receptors. ${ }^{27,31,47}$ Despite the fact that our team was fully aware of this crosstolerance effect, we decided to use low-frequency TENS (instead of high-frequency TENS) with our patient. This was motivated by two main reasons. First, contrarily to lowfrequency TENS (which produces a diffuse hypoalgesic effect), ${ }^{31,48}$ the effect of high-frequency TENS on pain is much more circumscribed, being limited to the region of the dermatome stimulated. ${ }^{49}$ Yet, using such segmental effect to decrease pain was hardly possible with our patient given that she could not tolerate TENS stimulations directly over her affected/allodynic lower leg. Second, the beneficial/ synergistic effect noted between tDCS and TENS by Boggio et al and Schabrun et al was observed for lowfrequency TENS. ${ }^{33,34}$

Regarding the long-term effects of our treatments on pain, as reported by the pain logbooks, the outcomes appear to be more favorable when tDCS is combined with TENS (Treatment B and C), as opposed to when tDCS is used alone (Treatment A). Nevertheless, it important to point out that pain reduction was modest and that we were able to see clinically important changes solely for the last intervention (Treatment C). ${ }^{45,46}$ Some elements could potentially explain the higher efficacy of tDCS combined with TENS when compared to tDCS alone. As hypothesized by Boggio et al and Schabrun et al, central neuronal plasticity mechanisms 
induced by tDCS and low-frequency TENS may have synergized when applied together. ${ }^{33,34}$ Our results are consistent with previous studies, including those of Boggio et al and Schabrun et al, in which the combination of these techniques seemed to have a synergetic effect in relieving pain of different etiologies, with effects lasting up to 3 months in some cases. ${ }^{33-35,39}$ In contrast, a study performed by our group on a small group of chronic pelvic pain patients showed no clinically significant effect on pain of a combined tDCS and TENS approach. ${ }^{42}$ It is probable that some chronic pain populations might be more responsive to this kind of approach than others.

The short time between our two first interventions (ie, tDCS alone and tDCS+TENS) surely limits our ability to differentiate the analgesic effect of tDCS alone from the potential synergetic effect of tDCS+TENS. Past studies have suggested that the hypoalgesic effect of tDCS and TENS could be cumulative, with individuals reporting greater pain relief with increasing number of sessions. ${ }^{21,50}$ Although we cannot rule out a cumulative effect of tDCS, the results obtained following the second tDCS+TENS intervention (Treatment $\mathrm{C}$, given 6 months later) somewhat argues in favor of a more potent effect of the combination of treatments, although both mechanisms could be simultaneously involved. The number of tDCS +TENS sessions required to optimally relieve pain is still unknown, but our observations could suggest that increasing the number of tDCS/TENS treatments could possibly lead to greater effects. ${ }^{21}$

The higher pain level noted during the pretreatment period of Treatment $\mathrm{C}$ could also have influenced our results. Still, the difference between the pretreatment pain intensity (Pre-Tx) of logbook 1 and logbook 2 are not clinically significant. ${ }^{4,46}$ As medication, SCS usage and life habits of the patient were maintained constant throughout the study, we believe that the variations in pretreatment pain between the logbooks can be considered as normal fluctuations.

Future studies should investigate the combined effects of tDCS and TENS using case control-series and randomized controlled trials with a larger sample size, including a TENS alone condition. We also recommend evaluating the aftereffect of the intervention on pain during a longer period, as our study shows lasting pain reduction effects that outlasted the 9-day follow-up. The influence of combined tDCS/ TENS treatments on physical function and quality of life, as well as the mechanisms involved in the cumulative effects, should also be investigated.

\section{Conclusion}

Patients suffering from CRPS, that are unresponsive to conventional treatment options, could potentially benefit from a combination of tDCS and TENS treatments to reduce pain. Non-invasive neurostimulation interventions, such as tDCS and TENS, could be interesting therapeutic strategies that could possibly be used more regularly with CRPS patients, given that randomized controlled trials are performed to confirm their efficacy in larger cohorts. Based on the case of our single patient, a modest pain reduction could be anticipated.

\section{Data Sharing Statement}

Authors will provide datasets supporting the results of this study upon demands of the editors.

\section{Consent for Publication}

The patient gave written informed consent for publication of this case report.

\section{Acknowledgments}

The authors wish to thank Marylène Ostiguy and Sébastien Alie for their help with data collection. We also wish to thank Johanie Dubé for her help with the manuscript.

\section{Funding}

GL is supported by the Fonds de recherche du QuébecSanté (FRQ-S).

\section{Disclosure}

The authors confirm that there are no known conflicts of interest associated with this publication.

\section{References}

1. Birklein F, Handwerker HO. Complex regional pain syndrome: how to resolve the complexity? Pain. 2001;94(1):1-6. doi:10.1016/S03043959(01)00393-1

2. Maihöfner C, Handwerker HO, Neundörfer B, Birklein F. Patterns of cortical reorganization in complex regional pain syndrome. Neurology. 2003;61(12):1707-1715. doi:10.1212/01.WNL.0000098939.02752.8E

3. Spicher C, Estebe J-P, Létourneau E, Packham TL, Rossier P, Annoni J-M. Diagnostic criteria for Complex Regional Pain Syndrome (CRPS). Douleur Analg. 2014;27:62-64. doi:10.1007/s11 724-014-0368-x

4. Rockett M. Diagnosis, mechanisms and treatment of complex regional pain syndrome. Curr Opin Anaesthesiol. 2014;27(5):494-500. doi:10.1097/ACO.0000000000000114

5. Birklein F, O’Neill D, Schlereth T. Complex regional pain syndrome: an optimistic perspective. Neurology. 2015;84(1):89-96. doi:10.1212/ WNL.0000000000001095

6. Harden RN. Objectification of the diagnostic criteria for CRPS. Pain Med. 2010;11(8):1212-1215. doi:10.1111/j.1526-4637.2010.00909.x 
7. Fairbank JC, Couper J, Davies JB, O'Brien JP. The Oswestry low back pain disability questionnaire. Physiotherapy. 1980;66(8):271-273.

8. Marinus J, Moseley GL, Birklein F, et al. Clinical features and pathophysiology of complex regional pain syndrome. Lancet Neurol. 2011;10(7):637-648. doi:10.1016/S1474-4422(11)70106-5

9. Harvey M, Maher-Bussières S, Emery E, et al. Evidence of motor system reorganization in complex regional pain syndrome type I: a case report. Can J Psychiatry. 2018;2(1):21-26. doi:10.1080/ 24740527.2017.1422116

10. O'Connell N, Wand B, McAuley J, Marston L, Moseley G. Interventions for treating pain and disability in adults with complex regional pain syndrome - an overview of systematic reviews. Cochrane Database Syst Rev. 2013;(4). doi:10.1002/14651858. CD009416.pub2.

11. Smart KM, Wand BM, O'Connell NE. Physiotherapy for pain and disability in adults with complex regional pain syndrome (CRPS) types I and II. Cochrane Database Syst Rev. 2016;2:CD010853. doi:10.1002/14651858.CD004158.pub3

12. Harden RN, Oaklander AL, Burton AW, et al. Complex regional pain syndrome: practical diagnostic and treatment guidelines, 4th edition. Pain Med. 2013;14(2):180-229. doi:10.1111/pme.12033

13. Turner-Stokes L, Goebel A, Group GD. Complex regional pain syndrome in adults: concise guidance. Clin Med (Lond). 2011;11 (6):596-600. doi:10.7861/clinmedicine.11-6-596

14. Perez RS, Zollinger PE, Dijkstra PU, et al. Evidence based guidelines for complex regional pain syndrome type 1. BMC Neurol. 2010;10:20. doi:10.1186/1471-2377-10-20

15. Goebel A. Management of adult patients with long-standing complex regional pain syndrome. Pain Manag. 2013;3(2):137-146. doi:10.22 17/pmt.13.2

16. Fregni F, Freedman S, Pascual-Leone A. Recent advances in the treatment of chronic pain with non-invasive brain stimulation techniques. Lancet Neurol. 2007;6(2):188-191. doi:10.1016/S14744422(07)70032-7

17. Mori $\mathrm{F}$, Codecà $\mathrm{C}, \mathrm{Kusayanagi} \mathrm{H}$, et al. Effects of anodal transcranial direct current stimulation on chronic neuropathic pain in patients with multiple sclerosis. J Pain. 2010;11(5):436-442. doi:10.1016/j.jpain. 2009.08.011

18. Ayache SS, Palm U, Chalah MA, et al. Prefrontal tDCS decreases pain in patients with multiple sclerosis. Front Neurosci. 2016;10:147. doi:10.3389/fnins.2016.00147

19. Plow EB, Pascual-Leone A, Machado A. Brain stimulation in the treatment of chronic neuropathic and non-cancerous pain. J Pain. 2012;13(5):411-424. doi:10.1016/j.jpain.2012.02.001

20. DosSantos MF, Love TM, Martikainen IK, et al. Immediate effects of tDCS on the $\mu$-opioid system of a chronic pain patient. Front Psychiatry. 2012;3:93.

21. David MCMM, Moraes AA, Costa MLD, Franco CIF. Transcranial direct current stimulation in the modulation of neuropathic pain: a systematic review. Neurol Res. 2018;40(7):555-563. doi:10.1080/ 01616412.2018.1453190

22. Nitsche MA, Paulus W. Excitability changes induced in the human motor cortex by weak transcranial direct current stimulation. $J$ Physiol. 2000;527 Pt 3:633-639. doi:10.1111/j.1469-7793.2000.t01-1-00633.x

23. Nitsche MA, Paulus W. Sustained excitability elevations induced by transcranial DC motor cortex stimulation in humans. Neurology. 2001;57(10):1899-1901. doi:10.1212/WNL.57.10.1899

24. Cruccu G, Garcia-Larrea L, Hansson P, et al. EAN guidelines on central neurostimulation therapy in chronic pain conditions. Eur J Neurol. 2016;23(10):1489-1499. doi:10.1111/ene.2016.23.issue-10

25. Bilgili A, Çakır T, Doğan Ş, Erçalık T, Filiz MB, Toraman F. The effectiveness of transcutaneous electrical nerve stimulation in the management of patients with complex regional pain syndrome: a randomized, double-blinded, placebo-controlled prospective study. J Back Musculoskelet Rehabil. 2016;29(4):661-671. doi:10.3233/ BMR-160667
26. Leonard G, Goffaux P, Marchand S. Deciphering the role of endogenous opioids in high-frequency TENS using low and high doses of naloxone. Pain. 2010;151(1):215-219. doi:10.1016/j.pain.2010.07.012

27. Léonard G, Cloutier C, Marchand S. Reduced analgesic effect of acupuncture-like TENS but not conventional TENS in opioid-treated patients. J Pain. 2011;12(2):213-221. doi:10.1016/j. jpain.2010.07.003

28. Bélanger A-Y. Transcutaneous electrical nerve stimulation. In: Wilkins LW, editor. Therapeutic Electrophysical Agents - Evidence Behind Practice. 3rd ed. Philadelphia, Baltimore, New York, London, Buenos Aires, Hong Kong, Sydney, Tokyo: Wolters Kluwer; 2015:256-280.

29. Choi JC, Kim J, Kang E, et al. Brain mechanisms of pain relief by transcutaneous electrical nerve stimulation: a functional magnetic resonance imaging study. Eur J Pain. 2015.

30. Marchand S. Chapter 1: applied pain neurophysiology. In: Pharmacology of Pain. Seattle: IASP Press; 2010:3-26.

31. Kalra A, Urban MO, Sluka KA. Blockade of opioid receptors in rostral ventral medulla prevents antihyperalgesia produced by transcutaneous electrical nerve stimulation (TENS). J Pharmacol Exp Ther. 2001;298(1):257-263.

32. Chipchase LS, Schabrun SM, Hodges PW. Corticospinal excitability is dependent on the parameters of peripheral electric stimulation: a preliminary study. Arch Phys Med Rehabil. 2011;92(9):14 23-1430. doi:10.1016/j.apmr.2011.01.011

33. Boggio PS, Amancio EJ, Correa CF, et al. Transcranial DC stimulation coupled with TENS for the treatment of chronic pain: a preliminary study. Clin J Pain. 2009;25(8):691-695. doi:10.1097/ AJP.0b013e3181af1414

34. Schabrun SM, Jones E, Elgueta Cancino EL, Hodges PW. Targeting chronic recurrent low back pain from the top-down and the bottom-up: a combined transcranial direct current stimulation and peripheral electrical stimulation intervention. Brain Stimul. 2014. doi:10.1016/j.brs.2014.01.058

35. Hazime FA, Baptista AF, de Freitas DG, et al. Treating low back pain with combined cerebral and peripheral electrical stimulation: a randomized, double-blind, factorial clinical trial. Eur J Pain. 2017;21(7):1132-1143. doi:10.1002/ejp.2017.21.issue-7

36. Flor H, Diers M. Sensorimotor training and cortical reorganization. NeuroRehabilitation. 2009;25(1):19-27. doi:10.3233/NRE-2009-0496

37. Moseley GL. Graded motor imagery for pathologic pain: a randomized controlled trial. Neurology. 2006;67(12):2129-2134. doi:10.1212/01.wnl.0000249112.56935.32

38. Léonard G, Mercier C, Tremblay LE. Effect of repetitive afferent electrical stimulation of the lower limb on corticomotor excitability and implications for rehabilitation. J Clin Neurosci. 2013;20 (3):435-439. doi:10.1016/j.jocn.2012.02.049

39. Meesen RL, Cuypers K, Rothwell JC, Swinnen SP, Levin O. The effect of long-term TENS on persistent neuroplastic changes in the human cerebral cortex. Hum Brain Mapp. 2011;32(6):872-882. doi:10.1002/hbm. 21075

40. Fraser C, Power M, Hamdy S, et al. Driving plasticity in human adult motor cortex is associated with improved motor function after brain injury. Neuron. 2002;34(5):831-840. doi:10.1016/S0896-6273(02)00705-5

41. Weiss C, Nettekoven C, Rehme AK, et al. Mapping the hand, foot and face representations in the primary motor cortex - retest reliability of neuronavigated TMS versus functional MRI. Neuroimage. 2013;66:531-542. doi:10.1016/j.neuroimage.2012.10.046

42. Harvey MP, Watier A, Dufort Rouleau É, Léonard G. Non-invasive stimulation techniques to relieve abdominal/pelvic pain: is more always better? World J Gastroenterol. 2017;23(20):3758-3760. doi:10.3748/wjg.v23.i20.3758

43. Chipchase LS, Schabrun SM, Hodges PW. Peripheral electrical stimulation to induce cortical plasticity: a systematic review of stimulus parameters. Clin Neurophysiol. 2011;122(3):456-463. doi:10.1016/j. clinph.2010.07.025 
44. Calvino B, Grilo RM. Central pain control. Joint Bone Spine. 2006;73(1):10-16. doi:10.1016/j.jbspin.2004.11.006

45. Farrar JT, Portenoy RK, Berlin JA, Kinman JL, Strom BL. Defining the clinically important difference in pain outcome measures. Pain 2000;88(3):287-294. doi:10.1016/S0304-3959(00)00339-0

46. Farrar JT, Young JP, LaMoreaux L, Werth JL, Poole RM. Clinical importance of changes in chronic pain intensity measured on an 11-point numerical pain rating scale. Pain. 2001;94(2):149-158. doi:10.1016/S0304-3959(01)00349-9

47. Sluka KA, Judge MA, McColley MM, Reveiz PM, Taylor BM. Low frequency TENS is less effective than high frequency TENS at reducing inflammation-induced hyperalgesia in morphine-tolerant rats. Eur J Pain. 2000;4(2):185-193. doi:10.1053/eujp.2000.0172
48. Facchinetti F, Sandrini G, Petraglia F, Alfonsi E, Nappi G, Genazzani AR. Concomitant increase in nociceptive flexion reflex threshold and plasma opioids following transcutaneous nerve stimulation. Pain. 1984;19(3):295-303. doi:10.1016/0304-3959(84) 90006-X

49. Ersek RA. Transcutaneous electrical neurostimulation: a new therapeutic modality for controlling pain. Clin Orthop Relat Res. 1977; (128):314-324.

50. Marchand S, Charest J, Li J, Chenard JR, Lavignolle B, Laurencelle L. Is TENS purely a placebo effect? A controlled study on chronic low back pain. Pain. 1993;54(1):99-106. doi:10.1016/ 0304-3959(93)90104-W

\section{Publish your work in this journal}

The Journal of Pain Research is an international, peer reviewed, open access, online journal that welcomes laboratory and clinical findings in the fields of pain research and the prevention and management of pain. Original research, reviews, symposium reports, hypothesis formation and commentaries are all considered for publication. The manuscript

Submit your manuscript here: https://www.dovepress.com/journal-of-pain-research-journal management system is completely online and includes a very quick and fair peer-review system, which is all easy to use. Visit http:// www.dovepress.com/testimonials.php to read real quotes from published authors. 